\title{
Security of multimodal dangerous freight transport: the way forward
}

\author{
G. L. L. Reniers \\ Antwerp Research Group on Safety and Security (ARGoSS), \\ University of Antwerp, Belgium \\ and
}

Centre for Economics and Corporate Sustainability (CEDON), HUB, KULeuven, Brussels, Belgium

\begin{abstract}
In this paper, we discuss the need for developing methodologies and tools for efficiently managing the taking of security countermeasures for hazmat transport. Transportation of dangerous freight is a complex problem characterized with multi-modality and with multiple stakeholders and decision-makers. Several future directions are given to advance academic and professional research in this ever more important field.
\end{abstract}

Keywords: hazmat transport, security, multi-modal transport, dangerous freight.

\section{Introduction}

Although until present the number of security-related accidents involving hazardous substances is rather limited in the Western World, a wide variety of security threats do exist against hazmat transport. A chemical shipment can fall victim to a number of different types of attacks inspired by terrorism. These types of attacks, combined with recent accidents, are changing the perception and tolerance of managing the risks of hazardous material transportation operations. The vulnerabilities of the transportation infrastructure and the potential consequences of hazmat transportation incidents urge policy makers to take action, resulting in worldwide calls for legislation directed at increased security, rerouting of hazardous material shipments with emphasis on security, and the push for application of inherently safer technologies, leading to enhanced 
security as a natural by-product. Public and private stakeholders fully agree upon the need for dangerous freight transport to be made safe and secure and to be organized as efficiently as possible.

Security-related risk assessment approaches or tools for hazmat transportation are hard to find. In fact, no generally accepted approach exists to assess security risks of moving hazardous substances. However, hazmat transport risks should be assessed with tools and approaches specifically designed to take the intentional nature of the risk, as well as moving risk features into account due to the specificity of such risk (Borysiewicz [1]). This is discussed more in depth hereafter.

Safety risks and security risks are to be identified, analyzed, assessed and evaluated in a different way. An assessment of safety risks requires consideration of (safety-related) scenarios, consequences, exposure to the risks, (quantitative) frequencies or probabilities, or (qualitative) likelihood. In case of an assessment of security risks, (security-related) scenarios, (qualitative) likelihood, consequences, threat, vulnerability, and target attractiveness are to be taken into account. It should be noted that target attractiveness is in function of time, political climate, etc., and can thus rapidly change.

For safety risks, so-called (safety) risk assessment methods are used. In case of security risks, so-called threat analyses or vulnerability assessments form part of (security) risk assessment methods. Fixed risks are also quite different from moving (transport-related) risks and taking security measures in case of hazmat transport often depends on the situation at hand. For example, with the exception of transport through pipeline, transported hazardous materials are not permanently present at a certain spatial point to perform transportation risk calculations; spatial planning around a transportation route can differ greatly from the surroundings of a fixed installation within a chemical plant; taking risk reducing measures is not always straightforward (e.g. an automatic sprinkler installation is very difficult to implement for hazmat transport, etc.); experts or specialists are not always present within a predefined period of time at a dangerous goods transport accident scene, etc.

Moreover, there are simply too many occurrences of hazmat transport to protect all of them in an equally efficient way. Hence, "Maginot Line" thinking should be abandoned and intelligent choices and decisions have to be made as regards which hazmat transport (which transport routes and which transport modes) need protection first, second, etc. The best defence against terrorist attacks on hazmat transport targets are adequate hazmat transport security vulnerability assessments and collaboration between and across the private sector, local, national and international cooperating authorities.

While hazardous materials accidents remain rare events $\left(10^{-8}-10^{-6}\right.$ per vehicle-mile) (Zografos and Davis [2]) and although the number of deaths and injuries due to all traffic accidents dwarfs the fatalities and injuries figures due to hazmat-related accidents, public concern about the risk of hazmat incidents is rather intense. Even minor incidents involving chemical freight strongly attract the attention of the general public, policy makers and industrialists. This is primarily due to the involuntary nature of the risk and the potential for 
significant consequences in case of such accidents. Despite the fact that there is genuine public concern about the risks associated with hazmat transportation systems, complete protection of these systems is economically and practically infeasible. Therefore, there is a need for a multi-disciplinary perspective on hazmat security, thereby taking the different transport modes into account, and based on a systems approach. Safety and security management specialists need to collaborate with transport (and other) experts, using e.g. the mathematical techniques of game-theory, operations research, etc. Such a holistic managerial approach should lead to more objective and cost-effective assessments and prioritizations of defenses and countermeasures against existing hazmat transportation threats.

At present, each year, close to 2 billion tons of hazardous materials are produced in the United States. The amount of hazardous materials shipments that are shipped each year is approximately 3 billion tons, since each shipment is moved several times before reaching its destination. In Europe, similar or even higher figures can be assumed. In China, since 2003, each year approximately a $25 \%$ rise of chemical industrial activities can be ascertained. Hazmat transport is thus essential for the world economy and their importance is continuously rising.

\section{Hazmat transportation and associated risks}

As indicated in the previous section, global trade has resulted in more worldwide national and transborder shipments of raw materials, dangerous goods, hazardous wastes, etc. than ever before. The volume of traffic and the speed with which the transport move continue to increase in both developed and developing countries. Remarkably, there have been relatively few intentional mass-casualty disasters associated with hazardous materials transport, especially when one considers the volumes carried per container, the number of shipments each year, and the direct proximity of the public. Historically, worldwide, only a very limited number of incidents causing mass casualties have happened, when expressed per billion ton-miles.

Until the present, the terrorist threat to the hazmat transportation system has gained far less attention from political and industrial policy makers than the passenger transport one, since very few terrorist organizations have made a serious attempt to either target hazmat transport, or to use hazmat transport as their means of attack in countries belonging to the Western world.

A recent and well-known security-related accident concerns a passenger train that was derailed by a powerful home-made bomb explosion on 28 November 2009 in Russia, thereby killing some 30 people and injuring some 100 people. Another security-related incident concerns an attempt to derail a passenger train in July 2010. In this sabotage incident, the railway was cut over 1.5 meters between Lelystad and Almere, two cities in The Netherlands. A well-known example of a security-related pipeline disaster happened in the Abule Egba district of Lagos, Nigeria. More than 260 people were killed and over 60 were injured after a gasoline pipeline exploded. The pipeline was ruptured deliberately and had been tapped by thieves for months prior to the accident. In another 
recent security-related pipeline accident on 11 August 2010, two people have been killed and one has been wounded after an oil pipeline exploded in southeast Turkey in an apparent terrorist attack by the PKK.

Terrorist actions may be targeted at obtaining as many human fatalities as possible, or at causing as much economic devastation as possible or they may be aimed at realizing both these objectives. It is important to note that under the present circumstances, the hazmat transportation system, in the USA as well as in Europe or any other part of the world, if under attack in an intelligent way, can be employed by terrorists to attain both these goals simultaneously.

Especially in the transportation system, escalation effects from an attack may be very far-reaching. As an example, in the Madrid terrorist attack on 11 March 2004, not only 191 people were killed and some 1,800 were injured, but the drop in the European markets (FTSE) following the Madrid bombings was calculated to be around $\$ 55$ billion. As another example, the estimated cost on the entire supply chain of a weapon of mass destruction shipped via container is some $\$ 1$ trillion (Zamparini [3]).

Therefore, to pro-actively limit the potential number of human fatalities and the huge economic costs, it is crucial that academic research is carried out from a multi-disciplinary, multi-modal and systemic viewpoint, to identify, to assess and to prioritize trans-border transportation system security risks, and to take pro-active actions and countermeasures accordingly.

It is obvious that research on security of hazmat transport from a multi-modal viewpoint deserves much more attention from private and public research institutes.

\section{How to improve security assessments of multi-modal dangerous freight transport}

One of the key factors of the chemical supply chain is multimodality; i.e., the use of different transport modes, each having their own characteristics with respect to economic and environmental parameters, as well as characterized with safety and security mode-specific features. Multimodal transportation is very much prevalent in the chemical industry, using road, railway, barges, ships, and pipelines to transport goods. A variety of companies are active usually within one of the available transport modi; and rarely do they operate within several of the modi at the same time. Nonetheless, by transporting chemical products employing different modes, (e.g. moving goods from road onto rail, ship or pipeline or vice versa, for security considerations), the chemical industry and its Logistics Service Providers can probably greatly improve their security scores.

Hence, the security resource allocation problem exists on both intra- and inter-modal levels. On an intra-modal dimension, the authorities or the companies responsible for the transport have to decide on which transportation routes (belonging to the same mode, that is road, railroad, inland waterways or pipeline) available between point A (e.g. a company, a city, or a storage park) and point B security measures have to be taken. This decision regarding security resources allocations should take into account all existing and feasible unimodal 
transportation routes between $\mathrm{A}$ and $\mathrm{B}$ and it should be repeated for all dangerous freight the authorities or the companies wish to investigate. On an inter-modal dimension, the different available modi for transporting certain dangerous freight between A and B should be investigated and security resources need to be allocated between the different modi, which forms an extra complexity to the unimodal security resources allocation problem. Actually, multimodal planning is more difficult than unimodal planning, both on an operational (i.e., planning of individual shipments), and on a tactical/strategic level (i.e., planning of flows of goods through the network). Therefore, an even stronger need for support by a transportation security risk model is present in the multi-modal case. Elaborating a user-friendly model and accompanying tool that supports decision-making by private as well as public stakeholders to allocate security resources from an intra-modal as well as an inter-modal viewpoint is thus very much needed.

In fact, the idea of developing a model or a tool addressing security-related issues and specifically aiming them at supporting decisions for preventing or mitigating intentional designed malicious acts on transportation infrastructure from a multi-modal perspective, has only very recently gained attention in the academic world.

Verma and Verter [4] are the first to examine population exposure due to hazmat transportation through intermodal service networks. In their 2010 paper, the same authors [5] suggest a lead-time based approach for planning rail-truck intermodal transportation of dangerous goods. Bell [6] shows that, for repeated hazardous materials shipments through a network, where the accident probabilities over the various links in the network are unknown, the safest strategy is in general to use multiple routes for each origin-destination pair. Bersani et al. [7] further demonstrate that spreading the risk both in space and in time further ameliorates the routing problem. Dadkar et al. [8] develop a gametheoretical model to represent the interaction of shipper/carrier and terrorist for hazmat transport and - by solving the model - give an idea of how routing decisions might be analyzed under terrorist threat. Garrido [9] presents mathematical models to identify possible hijacked vehicles' routes to vulnerable targets, assuming that probabilities of interception by law enforcement agents depend on the investment in defense resources. Murray-Tuite [10] demonstrates how Bayesian analysis can be used to update attack scenario probabilities after receiving new information on transportation risks.

However, to the best of the author's knowledge, no user-friendly multi-modal approach currently exists for assessing and prioritizing security risks (threats/vulnerabilities) related to the transportation of hazardous materials. The method should be understandable not only for academics, but also for both trained professionals as well as political decision makers, and the method should allow for simulation exercises (e.g. the user should be able to translate countermeasure improvements into the results of the threat assessment).

Different possibilities exist to meet with this need to develop a method for multi-modal assessment of hazmat transport. In any case, the approach implies the relative determination of hazmat transport or security countermeasures: 
different types of hazmat transport and/or various preventative and/or mitigation measures are relatively ranked towards each other. This way, one can obtain a notion of the relative (security related) vulnerability of transport routes and transport modes, thereby taking e.g. infrastructure characteristics and types and amounts of various transported chemicals into account. Using the obtained ranking results, a decision-maker can take more objective decisions on taking countermeasures for a certain hazmat transport route or can make a more neutral multi-modal choice of a hazmat transport route.

A first possibility concerns the use of multi-criteria analysis for developing and implementing a tool to deal with security of multi-modal hazmat transport.

The methodology should present a user-friendly approach to determine relative security risk levels of the different modes of hazardous freight transport (i.e., road, railway, inland waterways and pipeline transportation), taking into account various possible countermeasures. Likelihood parameters of securityrelated accidents in which dangerous freight is involved and possibly causing fatalities in the surrounding population should be considered, together with the consequences of accident scenarios, to determine transportation route security risk levels. Such an approach should lead to a multi-modal user-friendly security threat assessment tool which can be used by policy makers as well as by industrialists (shippers or Logistics Service Providers). The generic method would allow for comparing the security risk levels of the different routes of transportation of hazardous goods and for taking countermeasures from a unimodal as well as from a multi-modal perspective.

A second possibility involves the development and the use of a gametheoretical model and tool.

Since the terrorist attacks on 11 September 2001, game theory has been increasingly employed as a mathematical tool to deal with security decisions facing with adaptive adversaries. In particular, recent literature includes Bier [11], Bier et al. [12], Zhuang and Bier [13]; Heal and Kunreuther [14]; Paruchuri et al. [15]; Bier et al. [16]; Dighe et al. [17]; Guikema and Aven [18]; and Zhuang et al. [19]. See Sandler and Siqueira [20] for an extensive survey. More specifically, gaining insights into the nature of optimal defensive investments yielding the best trade-off between investment costs and critical infrastructure security was also already subject of an important amount of scientific research, e.g., Brown et al. [21]; Azaiez and Bier [22]; Levitin [23]; Patterson and Apostolakis [24]; Levitin and Hausken [25]; Hausken [26]; Liu et al. [27]; Hausken and Levitin [28]; Golany et al. [29]; Reniers [30].

However, to date, no concrete attention has been paid to the multimodal transportation security resources allocation decision problem from a gametheoretical viewpoint. This is thus a research subject deserving much more attention from the academia. In case of the multi-modal transportation problem, the focus should be on the development of an attacker-defender model (based on game theory). A dynamic game with incomplete information can be considered in which the defender chooses how to allocate the security resources (on which transport routes, on which modes, etc.), and then an attacker chooses which target to attack (which route, which mode, etc.) according to a multi-attribute 
utility function. A model focusing on multi-modal transportations of hazardous substances should make the decision process of taking security countermeasures allocations in a complex transportation network more objective and (subsequently) more justified.

Cox [31] indicates that game theory and risk analysis are mutually reinforcing in order to obtain effective risk management recommendations for allocating security resources. Therefore, the use, by private as well as public stakeholders, of both a tool based on multi-criteria analysis and one based on game-theory, might lead to a truly more secured hazmat transportation system.

\section{Conclusions}

As the amounts of dangerous goods transported continue to rise in both developed and developing countries, and as population characteristics continue to increase throughout the world, and the distance between the public and the hazardous materials transportation operations continuously diminishes, controlling and managing risks within the multi-modal hazardous materials transportation system becomes ever more important for industrialists and policy makers. Especially risks associated with intentional acts are subject of the emerging research field devoted to the hazmat transportation system.

From a multi-disciplinary researchers' perspective as well as from a policy makers' viewpoint, the transportation system security research area needs to be treated as a mature and extremely important domain with a direct impact on the global economic market.

Methodologies and accompanying software need to be developed to deal with the complex multi-modal hazmat transportation security brain-teaser and the security resources allocation problem. Two possible mutually reinforcing ways for advancing multi-modal hazmat security, are represented by multi-criteria analysis on the one hand and game-theory on the other hand. Both these techniques are very promising to help tackling hazmat transport security of the next generation.

\section{References}

[1] Borysiewicz M.J., 2006. Transportation Risk Assessment, Report IAE B54/2006, Institute of Atomic Energy.

[2] Zografos, K., Davis, C., 1989. Multi-objective programming approach for routing hazardous materials. Journal of Transportation Engineering, 115, 661-673.

[3] Zamparini, L., 2010. Transport Security in EU and US: Competing or Complementary visions? Nectar workshop on transport security, Lecce, Italy, 5-6 Feb 2010.

[4] Verma, M., Verter, V., 2007. Railroad transportation of dangerous goods: Population exposure to airborne toxins. Computers and Operations Research, 34, 1287-1303. 
[5] Verma, M., Verter, V., 2010. A lead-time based approach for planning railtruck intermodal transportation of dangerous goods. European Journal of Operational Research, 202, 696-706.

[6] Bell, M.G.H., 2006. Mixed route strategies for the risk-averse shipment of hazardous materials. Networks and Spatial Economics, 6, 253-265.

[7] Bersani, Ch., Minciardi, R., Tomasoni, A.M., Sacile, R., 2009. Risk Averse Routing of Hazardous Materials with Scheduled Delays. In: Security and Environmental Sustainability of Multimodal Transport, Bell, M.G.H., Hosseinloo, S.H., Kanturska, U. (Edits.), NATO Science for Peace and Security Series - C: Environmental Security, Springer, Dordrecht, The Netherlands

[8] Dadkar, Y., Nozick, L., Jones, D., 2009. Routing of Hazardous Material Shipments Under the Threat of Terrorist Attack, In: Security and Environmental Sustainability of Multimodal Transport, Bell, M.G.H., Hosseinloo, S.H., Kanturska, U. (Edits.), NATO Science for Peace and Security Series - C: Environmental Security, Springer, Dordrecht, The Netherlands.

[9] Garrido, R.A., 2009. Terrorists and Hazmat: A Methodology to Identify Potential Routes, In: Security and Environmental Sustainability of Multimodal Transport, Bell, M.G.H., Hosseinloo, S.H., Kanturska, U. (Edits.), NATO Science for Peace and Security Series - C: Environmental Security, Springer, Dordrecht, The Netherlands.

[10] Murray-Tuite, P., 2009. Bayesian analysis for transportation risk, In: Security and Environmental Sustainability of Multimodal Transport, Bell, M.G.H., Hosseinloo, S.H., Kanturska, U. (Edits.), NATO Science for Peace and Security Series - C: Environmental Security, Springer, Dordrecht, The Netherlands.

[11] Bier, V.M., 2007. Choosing what to protect. Risk Analysis, 27(3), 607-620.

[12] Bier, V.M., Oliveros S., Samuelson L. 2007. Choosing what to protect: Strategic defensive allocation against an unknown attacker. Journal of Public Economic Theory 9, 563-587.

[13] Zhuang, J. and Bier, V.M. 2007. Balancing Terrorism and Natural Disasters-Defensive Strategy with Endogenous Attack Effort. Operations Research 55(5), 976-991.

[14] Heal, G., Kunreuther, H., 2007. Modeling interdependent risks. Risk Analysis, 27(3), 621-634.

[15] Paruchuri, P., Pearce, J.P., Marecki, J., Tambe, M., Ordonez, F., Kraus, S., 2008. Efficient algorithms to solve Bayesian Stackelberg games for security applications. AAA'08: Proceedings of the 23rd national conference on artificial intelligence, vol. 3, AAAI Press, 1559-1562.

[16] Bier, V.M., Haphuriwat, N., Menoyo, J., Zimmerman, R. and Culpen, A. 2008. Optimal Resource Allocation for Defense of Targets Based on Differing Measures of Attractiveness. Risk Analysis 28(3), 763-770.

[17] Dighe, N., Zhuang J., and Bier V.M., 2009. Secrecy in defensive allocations as a strategy for achieving more cost-effective attacker deterrence, International Journal of Performability Engineering, special 
issue on System Survivability and Defense against External Impacts, 5(1): 31-43.

[18] Guikema, S.D., Aven, T., 2010. Assessing risk from intelligent attacks: A perspective on approaches. Reliability Engineering and System Safety, 95, 478-483.

[19] Zhuang, J., Bier, V.M., and Alagoz, O. 2010. Modeling Secrecy and Deception in a Multiple-period Attacker-Defender Signaling Game, European Journal of Operational Research, 203(2): 409-418.

[20] Sandler, T. and Siqueira, K. 2009. Games and Terrorism. Simulation \& Gaming 40, 2, 164-192.

[21] Brown, G., Carlyle, M., Salmeron, J. and Wood, K. 2006. Defending Critical Infrastructure. Interfaces 36, 530-544.

[22] Azaiez, N., Bier, V.M., 2007. Optimal Resource Allocation for Security in Reliability Systems. European Journal of Operational Research, 181(2), 773-786.

[23] Levitin, G., 2007. Optimal Defense Strategy Against Intentional Attacks. IEEE Transactions on Reliability 56(1), 148-156.

[24] Patterson, S. Apostolakis, G. 2007, Identification of critical locations across multiple infrastructures for terrorist actions, Reliability Engineering \& System Safety, 92(9), 1183-1203.

[25] Levitin, G. and Hausken, K. 2008. Protection vs. Redundancy in Homogeneous Parallel Systems. Reliability Engineering \& System Safety, 93(10), 1444-1451.

[26] Hausken, K., 2008. Strategic defense and attack for reliability systems. Reliability Engineering and System Safety, 93(11), 1740-1750.

[27] Liu, D., Wang, X., Camp, J., 2008. Game-theoretic modeling and analysis of insider threats. International Journal of Critical Infrastructure Protection, 1.

[28] Hausken, K., Levitin, G., 2009. Minmax defense strategy for complex multi-state systems. Reliability Engineering and System Safety, 94(2), 577587.

[29] Golany, B., Kaplan, E.H., Marmur, A., Rothblum, U.G., 2009. Nature plays with dice - terrorists do not: allocating resources to counter strategic versus probabilistic risks. European Journal of Operational Research, 192(1), 198-208.

[30] Reniers, G., 2010. An external domino effects investment approach to improve cross-plant safety within chemical clusters. Journal of Hazardous Materials, 177(1-3), 167-174.

[31] Cox, L.A., 2009. Game Theory and Risk Analysis. Risk Analysis, 29(8), 1062-1068. 